\title{
Preparation of a dual cored hepatoma-specific star glycopolymer nanogel via arm-first ATRP approach
}

\author{
This article was published in the following Dove Press journal: \\ International Journal of Nanomedicine \\ II May 2017 \\ Number of times this article has been viewed
}

\author{
Shaofeng Lou' \\ Xiuyuan Zhang ${ }^{2}$ \\ Mingming Zhang ${ }^{2}$ \\ Shenglu $\mathrm{ji}^{\prime}$ \\ Weiwei Wang ${ }^{2}$ \\ Ju Zhang' \\ Chen $\mathrm{Li}^{2}$ \\ Deling Kong ${ }^{1,2}$
}

'Key Laboratory of Bioactive Materials, Ministry of Education, College of Life Sciences, Nankai University, ${ }^{2}$ Tianjin Key Laboratory of Biomaterial Research, Institute of Biomedical Engineering, Chinese Academy of Medical Science, Tianjin, People's Republic of China
Correspondence: Deling Kong Institute of Molecular Biology, Nankai University, Tianjin 30007I, People's Republic of China

Tel +86 222350 I 229

Fax +862223498775

Email kongdeling@nankai.edu.cn

Chen Li

Tianjin Key Laboratory of Biomaterial Research, Institute of Biomedical Engineering, Chinese Academy of Medical Science, No 236, Baidi Road, Tianjin 300192 , People's Republic of China $\mathrm{Tel} / \mathrm{fax}+862287893696$

Email cli0616826@I26.com
Abstract: A reductase-cleavable and thermo-responsive star-shaped polymer nanogel was prepared via an "arm-first" atom transfer radical polymerization approach. The nanogel consists of a thermo- and redox-sensitive core and a zwitterionic copolymer block. The dual sensitive core is composed of poly(N-isopropylacrylamide) that is formed by disulfide crosslinking of $\mathrm{N}$-isopropylacrylamide. The zwitterionic copolymer block contains a poly(sulfobetaine methacrylate) component, a known anti-adsorptive moiety that extends blood circulation time, and a lactose motif of poly(2-lactobionamidoethyl methacrylamide) that specifically targets the asialoglycoprotein receptors (ASGP-Rs) of hepatoma. Doxorubicin (DOX) was encapsulated into the cross-linked nanogels via solvent extraction/evaporation method and dialysis; average diameter of both blank and DOX-loaded nanogels was $\sim 120 \mathrm{~nm}$. The multi-responsiveness of nanogel drug release in different temperatures and redox conditions was assessed. After $24 \mathrm{~h}$, DOX release was only $\sim 20 \%$ at $30^{\circ} \mathrm{C}$ with $0 \mathrm{mM}$ glutathione (GSH), whereas over $90 \%$ DOX release was observed at $40^{\circ} \mathrm{C}$ and $10 \mathrm{mM} \mathrm{GSH}$, evidence of dual responsiveness to temperature and reductase GSH. The $\mathrm{IC}_{50}$ value of DOX-loaded nanogels was much lower in human hepatoma (HepG2) cells compared to non-hepatic HeLa cells. Remarkably, DOX uptake of HepG2 cells differed substantially in the presence and absence of galactose $(0.31 \mathrm{vs} 1.42 \mu \mathrm{g} / \mathrm{mL}$ after $48 \mathrm{~h}$ of incubation). The difference was non-detectable in HeLa cells ( $1.21 \mathrm{vs} 1.57 \mu \mathrm{g} / \mathrm{mL}$ after $48 \mathrm{~h}$ of incubation), indicating that the overexpression of ASGP-Rs leads to the DOX-loaded lactosylated nanogels actively targeting hepatoma. Our data indicate that the lactose-decorated star-shaped nanogels are dual responsive and hepatoma targeted, and could be employed as hepatoma-specific anti-cancer drug delivery vehicle for cancer chemotherapy.

Keywords: glycopolymer, multi-responsive nanogel, hepatoma targeting, drug delivery, armfirst ATRP

\section{Introduction}

Extensive efforts have been made in nanomedicine development in order to improve drug loading efficiency and controlled drug release in response to environmental stimuli with reduced systemic toxicity. ${ }^{1,2}$ In the past decade, research within the fields of nanomedicine has demonstrated that nanodrug delivery systems could substantially improve drug bioavailability and local concentration by preventing premature degradation, controlled release, and enhanced permeability and retention effect. ${ }^{3,4}$ However, rapid systemic elimination and unspecific and burst release are still major concerns that limit clinical potential of nanomedicine. To optimize nanodrug delivery system, design, and development of multivalent nanoparticles by surface conjugation of bioactive targeting moieties, stimuliresponsive monomers and anti-adsorptive copolymer blocks have been reported. ${ }^{5-10}$ 
Star polymer nanogels, also termed smart drug delivery systems, have shown potential as efficient diagnostic and/or therapeutic tools against cancer, infectious diseases, or neurodegenerative disorders. ${ }^{11-13}$ Indeed, it has been reported that star-shaped polymer nanogels prepared by controlled/“living" radical polymerization are, in principle, suitable for delivery of multi-functional molecular cargos (eg, drugs, gene, and imaging agents), controlling cellular uptake, and triggering intracellular release. ${ }^{14}$ In addition, star polymer nanogels also exhibit stable physicochemical properties, which are responsible for their prolonged systemic retention. ${ }^{15}$ Moreover, star polymer nanogels are often composed of a hydrophobic core formed by polymer cross-linkage and hydrophilic "arms". This unique architecture enables high drug loading capacity due to hydrophobic interaction between drugs and the core, and the "arms" allow extensive modifications with chemical and bioactive ligands for active and targeted delivery. A wide range of bioactive ligands including proteins and their bioactive fragments, short-chain peptides, nucleic acid aptamers, folic acid, and sugar molecules have been selected for nanogel modifications. ${ }^{5}$ Glycosylation is one of the promising approaches for active targeted delivery due to the inherent biocompatibility and bioadhesiveness of sugar molecules and the pivotal roles of carbohydrate recognition mechanisms in major biological events. ${ }^{16}$ Studies have reported functionalization with terminal fructopyranose moieties for breast cancer cells targeting, ${ }^{17}$ and mannose as specific ligand for immune scavenge cells such as macrophages and dendritic cells..$^{18,19}$

Moreover, glycosylated nanocarriers with terminal galactose or lactose moieties have also shown selectivity for hepatic cells, with clinical relevance for hepatoma chemotherapy or hepatitis $\mathrm{C}$ treatment. ${ }^{20}$ Indeed, hepatoma, the most common type of human cancer, is known to express abnormally high level of the asialoglycoprotein receptors (ASGP-Rs) that are responsive to carbohydrate motifs including the $\mathrm{N}$-acetyl galactosamine, galactose, and lactose. ${ }^{4,6,10,21-25}$ It is also noteworthy that malignant tumor cells also elicit changes in the stroma for rapid growth and progression, thus creating an abnormal microenvironment characterized by hypoxia, acidity, and overexpression of surface receptors and enzymes. ${ }^{1,4,9}$ Given the distinct tumor microenvironment, designing polymeric micelles that are responsive to redox reaction, $\mathrm{pH}$, and temperature changes to facilitate tumor-specific drug release has been an effective tumor-targeted drug delivery approach. In particular, the utilization of disulfide-containing reversible cross-linkers has gained ample research attention, owing to the fact that the disulfide bond is reducible and can be cleaved by reductase such as glutathione (GSH), an endogenous anti-oxidant that could translocate to the cell surface. ${ }^{24,26}$

Considering the targeting ability of glycosylation and the unique characteristics of tumor microenvironment, a reductase-cleavable and thermo-responsive star-shaped polymer nanogel was prepared via an "arm-first" atom transfer radical polymerization (ATRP) approach for targeted hepatoma drug delivery (Scheme 1). The thermo- and redox-sensitive core is composed of disulfide bonds (-S-S-) cross-linked poly(N-isopropylacrylamide) (PNIPAM), which is conjugated with arms formed of anti-adsorptive zwitterionic copolymer block and poly(sulfobetaine methacrylate) (PSBMA), to minimize interactions of the polymeric nanogels with plasma protein and cells. A lactose moiety, poly(2-lactobionamidoethyl methacrylamide) (PLAMA), was also included as targeting segment that recognizes hepatocellular ASGP-Rs. Characteristics of the star-shaped nanogels were assessed by nuclear magnetic resonance (NMR) and transmission electron microscope (TEM). Drug releasing property in response to thermo and redox changes was assessed using DOX as a model anti-cancer drug. Cytotoxicity, drug uptake, and intracellular releasing kinetics of the DOX-loaded nanogels were evaluated in human hepatocellular cancer cells (HepG2) and non-hepatic cervical cancer cells (HeLa) as control.

\section{Material and methods Chemicals}

Doxorubicin hydrochloride (DOX. $\mathrm{HCl})$ was purchased from Beijing Huafeng United Technology Co., Ltd. Lactobionic acid, 2-aminoethyl methacrylate hydrochloride, and $\mathrm{N}, \mathrm{N}^{\prime}$-bis(acryloyl)cystamine (BAC) were purchased from Sigma-Aldrich. N-isopropylacrylamide (NIPAM; Sinopharm Chemical Reagent Co., Ltd) was recrystallized from an n-hexane/toluene mixture. $\mathrm{CuBr}$ (Tianjin, People's Republic of China, analytical reagent) was purified by dissolving in concentrated $\mathrm{HCl}$, precipitating in water, washing with ethanol and ethyl ether three times, and then drying under vacuum. The following reagents were of analytic grade and used as received: 2-bromoisobutyryl bromide (Tokyo Chemical Industry), dithiothreitol (Acros Organics), 2,2'-bipyridine (bpy 99\%, Sigma-Aldrich), and triethylamine (Sigma-Aldrich). 2-Lactobionamidoethyl methacrylamide (LAMA) was synthesized using a method described elsewhere. ${ }^{27,28}$ Water was purified by a Milli-Q system (Millipore Corporation) to a specific resistivity of $\sim 18 \mathrm{M} \Omega$ $\mathrm{cm}$. All solvents were distilled and dried by storing over activated $4 \AA$ molecular sieves for $24 \mathrm{~h}$ prior to use. 
<smiles>O=C(O)C(O)C(O)C(O)C(O)C(O)C(O)C(O)CO</smiles><smiles>C=C(C)C(=O)OCCN</smiles>

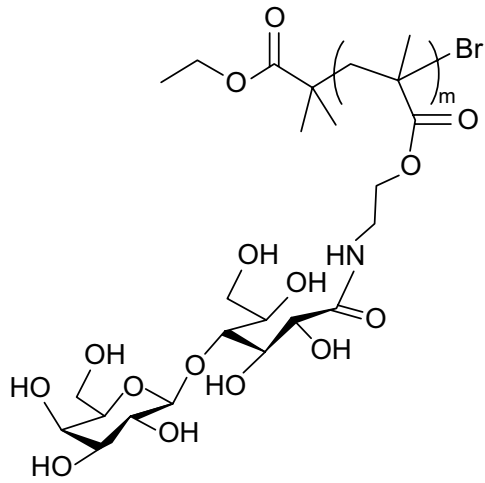

PLAMA-Br

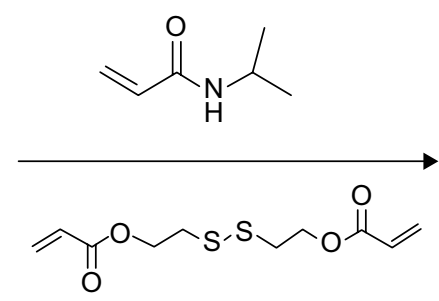

CuBr, bpy, Span $80,50^{\circ} \mathrm{C}, 48 \mathrm{~h}$
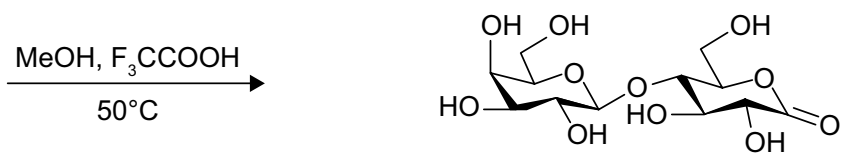

Lactobionolactone

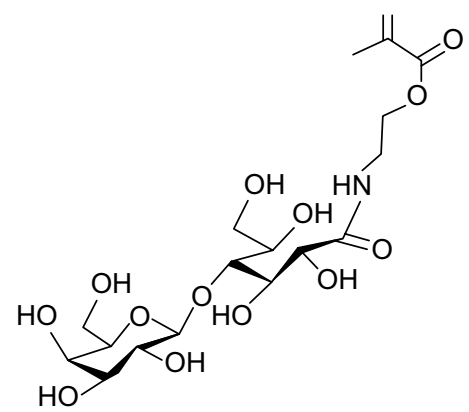

LAMA

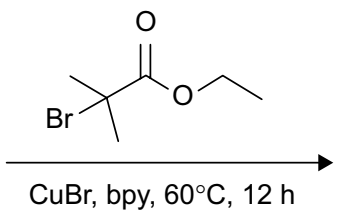

CuBr, bpy, $60^{\circ} \mathrm{C}, 12 \mathrm{~h}$

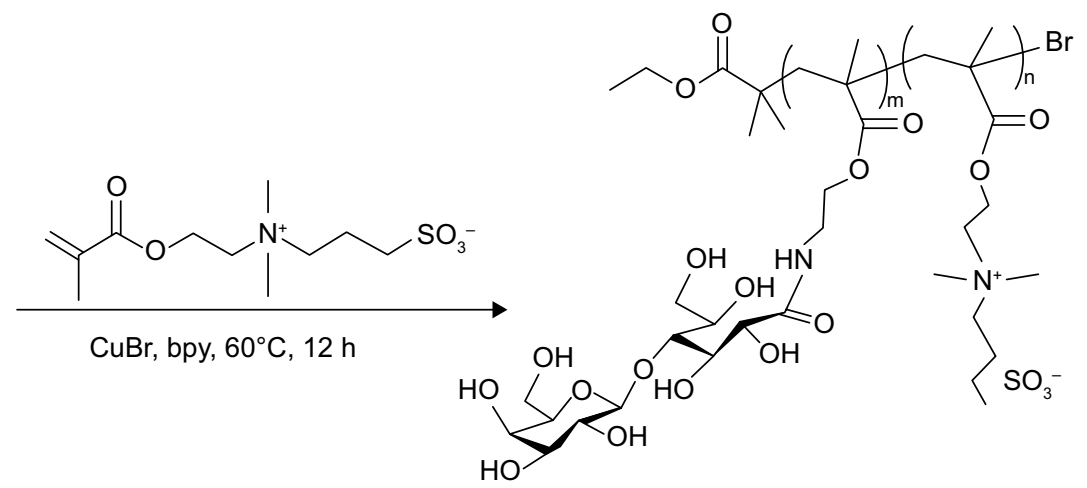

PLAMA-b-PSBMA-Br

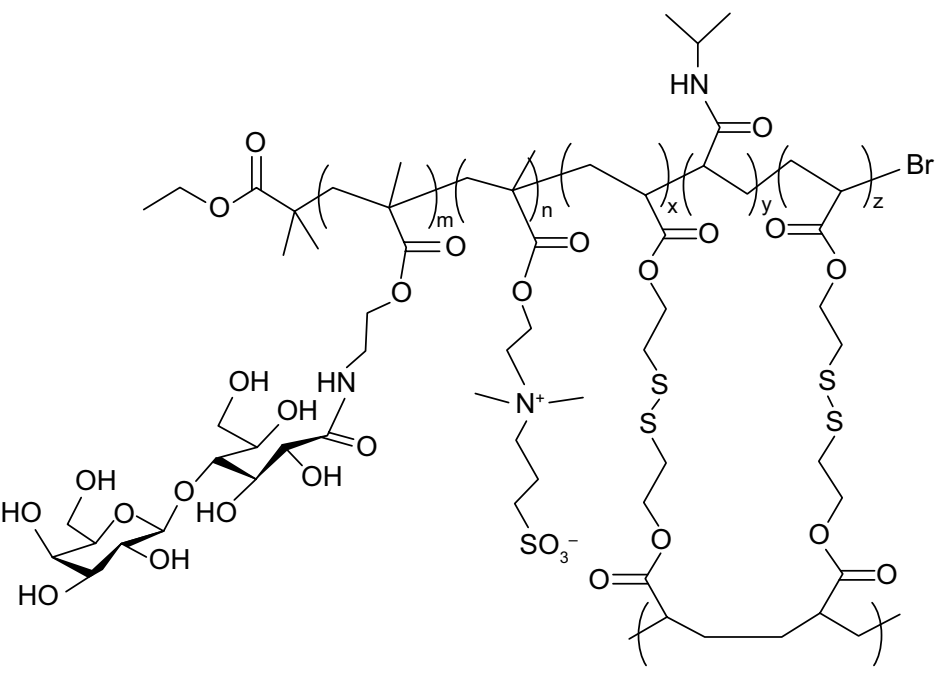

s-(PLAMA-b-PSBMA)-b-PNIPAM nanogels

Scheme I The synthesis route of the star nanogels.

\section{Synthesis of LAMA}

LAMA was synthesized as reported before. ${ }^{27,28}$ Briefly, lactobionic acid was dissolved in anhydrous methanol at $50^{\circ} \mathrm{C}$ with aliquot trifluoroacetic acid as catalyst, followed by vacuum distillation. Lactobionolactone (10.0 g, $29.4 \mathrm{mmol})$ was first dissolved in methanol at $40^{\circ} \mathrm{C}$ and then cooled to room temperature before the addition of 2-aminoethyl methacrylate hydrochloride $(10.0 \mathrm{~g}, 60.4 \mathrm{mmol})$, triethylamine 
(10.0 mL), and hydroquinone (0.25 g). The mixture was stirred for $5 \mathrm{~h}$, concentrated by rotary evaporation, and precipitated into either 2-propanol or dichloromethane. ${ }^{1} \mathrm{H}$ NMR spectrum of LAMA monomer is represented in Figure 1. The white solid formed was filtered, washed with 2-propanol, dried under vacuum, and isolated in $72 \%$ yield.

\section{Synthesis of PLAMA macroinitiator}

PLAMA macroinitiator (PLAMA-Br) was prepared by ATRP with $\mathrm{CuBr}$ complexed by bpy as the catalyst in DMF at $60^{\circ} \mathrm{C}$. Copper(I) bromide $(0.012 \mathrm{~g}, 0.085 \mathrm{mmol})$ and two equiv of bpy (0.026 g, $0.170 \mathrm{mmol})$ were first dissolved in dry DMF $(5 \mathrm{~mL})$ in a $10 \mathrm{~mL}$ two-neck flask and degassed with two freeze-pump-thaw cycles. 2-Bromoisobutyryl bromide $\left(0.028 \mathrm{~g}, 0.142 \mathrm{mmol}\right.$, target $\left.\mathrm{DP}_{\mathrm{n}}=30\right)$ and LAMA $(2.0 \mathrm{~g}, 4.26 \mathrm{mmol})$ were dissolved in dry DMF $(3.5 \mathrm{~mL})$ in a $10 \mathrm{~mL}$ two-neck flask and degassed with two freeze-pumpthaw cycles. Then the solution was added into the solution through a syringe under nitrogen atmosphere, and the mixture was degassed with another three freeze-pump-thaw cycles. The reaction mixture was then stirred at $60^{\circ} \mathrm{C}$ for $12 \mathrm{~h}$. The resulting dispersions were dialyzed (cutoff 3,500 Da) against water for $48 \mathrm{~h}$ to ensure complete removal of unreacted monomers and recovered by lyophilization.

\section{Synthesis of zwitterionic diblock glycopolymer PLAMA-b-PSBMA-Br with PLAMA macroinitiator}

Copper(I) bromide $(0.021 \mathrm{~g}, 0.147 \mathrm{mmol})$ and two equiv of bpy $(0.045 \mathrm{~g}, 0.294 \mathrm{mmol})$ were first dissolved in dry DMF $(5 \mathrm{~mL})$ in a $10 \mathrm{~mL}$ two-neck flask and degassed with two freeze-pump-thaw cycles. PLAMA-Br (1.3 g, 0.098 mmol) and SBMA (0.55 g, $1.97 \mathrm{mmol})$ were dissolved in DMF and Milli-Q water mixture (3:5) in a $10 \mathrm{~mL}$ two-neck flask and degassed with two freeze-pump-thaw cycles. Then the solution was added into the solution through a syringe under nitrogen atmosphere, and the mixture was degassed with another three freeze-pump-thaw cycles. The reaction mixture was then stirred at $60^{\circ} \mathrm{C}$ for $12 \mathrm{~h}$. The resulting dispersions were dialyzed (cutoff 8,000 Da) against water for $48 \mathrm{~h}$ to ensure complete removal of unreacted monomers and recovered by lyophilization.

\section{Preparation of zwitterionic triblock star-shaped glycopolymer s-(PLAMA-b- PSBMA)-b-PNIPAM nanogels}

Copper(I) bromide ( $0.021 \mathrm{~g}, 0.147 \mathrm{mmol})$ and two equiv of bpy $(0.045 \mathrm{~g}, 0.294 \mathrm{mmol})$ were first dissolved in dry DMF
$(5 \mathrm{~mL})$ in a $10 \mathrm{~mL}$ two-neck flask and degassed with two freeze-pump-thaw cycles. PLAMA-b-PSBMA-Br (1.6 g, $0.098 \mathrm{mmol}), \mathrm{BAC}(0.5 \%-2 \%$ molar ratio), NIPAM (0.331 g, $2.99 \mathrm{mmol})$, and Span $80(0.10 \mathrm{~g})$ were dissolved in DMF and water mixture (2:5) in a $10 \mathrm{~mL}$ two-neck flask and degassed with two freeze-pump-thaw cycles. Then the solution was added into the solution through a syringe under nitrogen atmosphere, and the mixture was degassed with another three freeze-pump-thaw cycles. The reaction mixture was then stirred at $50^{\circ} \mathrm{C}$ for $12 \mathrm{~h}$. The resulting dispersions were dialyzed (cutoff 8,000 Da) against water for $48 \mathrm{~h}$ to ensure complete removal of unreacted monomers and recovered by lyophilization.

\section{Characterization}

${ }^{1} \mathrm{H}$ NMR was performed on a Bruker $400 \mathrm{MHz}$ spectrometer using $\mathrm{D}_{2} \mathrm{O}$ as solvent. Molecular weights $\left(\mathrm{M}_{\mathrm{w}}\right.$ and $\left.\mathrm{M}_{\mathrm{n}}\right)$ and molecular weight distributions (polydispersity index, PDI $\left.=M_{w} / M_{n}\right)$ of s-(PLAMA-b-PSBMA)-b-PNIPAM were determined by gel permeation chromatography (GPC) measurements on a Waters LS measurement system using water as the mobile phase, at a flow rate of $1.0 \mathrm{~mL} / \mathrm{min}$ with a column temperature of $35^{\circ} \mathrm{C}$. The optical absorption of the s-(PLAMA-b-PSBMA)-b-PNIPAM aqueous solutions $(1 \mathrm{mg} / \mathrm{mL})$ was measured at $550 \mathrm{~nm}$ with a PerkinElmer Lambda $35 \mathrm{UV}$-vis spectrometer, equipped with a multicell thermoelectric temperature controller $\left( \pm 0.1^{\circ} \mathrm{C}\right)$. The temperature was increased from $10^{\circ} \mathrm{C}$ to $45^{\circ} \mathrm{C}$ at $1^{\circ} \mathrm{C} / 5 \mathrm{~min}$, and absorption recorded at each temperature. The upper critical solution temperature (UCST) or lower critical solution temperature (LCST) in a particular solution condition was the midpoint of the temperature absorption curve. ${ }^{29,30}$ The average hydrodynamic diameter of the s-(PLAMA-b-PSBMA)-b-PNIPAM in water was measured by dynamic light scattering (DLS) (Brookhaven BI-200SM goniometer) equipped with a solid state laser source emitting at $532 \mathrm{~nm}$ and fitted with an external water bath and thermostat. The volume phase transition temperatures of the nanogels were examined by microcalorimetric analyses.

For encapsulation of DOX into the nanogels, dried nanogels $(10.0 \mathrm{mg})$ were allowed to stir in water $(20 \mathrm{~mL})$ for 2 days to become fully swollen and then mixed with DOX (2.0 mg). The resulting reddish mixture was stirred for $24 \mathrm{~h}$, and then transferred to a dialysis tube $\left(\mathrm{M}_{\mathrm{w}}=3,400 \mathrm{kDa}\right)$ in aqueous. Unloaded DOX was removed by extensive dialysis in water. Drug loading content (LC) and encapsulation efficiency were measured with UV-vis spectrophotometer (UV-2550; Shimadzu, Kyoto, Japan). The morphology change of 
microgels before and after adding $10 \mathrm{mM}$ with an intracellular level within millimolar range $(1-10 \mathrm{mM})$ and extracellular concentration typically at micromolar $(20-40 \mu \mathrm{M})$ level was measured by TEM (JEOL, JSM-7500F) for $4 \mathrm{~h}$.

\section{In vitro drug release assay}

In vitro drug release experiments were performed as following: $10 \mathrm{mg}$ of freeze-dried DOX-loaded nanogels with $2 \mathrm{~mL}$ phosphate-buffered saline (PBS) buffer was placed into a dialysis bag (MWCO 3500). Then the release system was suspended in $10 \mathrm{~mL}$ of different temperature and aliquot GSH in PBS solution. After a predetermined period, $2 \mathrm{~mL}$ of solution was taken from release system for analysis, and $2 \mathrm{~mL}$ of fresh medium was added into the release buffer. The DOX concentration of each aliquot was determined by UV-vis analysis at $480 \mathrm{~nm}$. All release experiments were carried out in triplicate. The release percentage of DOX was calculated using Eq (1):

$$
\text { Drug release }(\%)=\frac{M_{t}}{M_{\infty}} \times 100 \text {, }
$$

where $M_{t}$ is the amount of DOX released from the nanogels at time $t$ and $M_{\infty}$ is the amount of the DOX loaded in the nanogels.

\section{In vitro cytotoxicity assay}

Cytotoxicity of DOX-s-(PLAMA-b-PSBMA)-b-PNIPAM was assessed using a cell counting kit-8 (CCK-8). The human hepatoma HepG2 and cervical carcinoma HeLa cell lines were purchased from Type Culture Collection of the Chinese Academy of Sciences, Shanghai, People's Republic of China. Briefly, human hepatoma HepG2 and cervical carcinoma HeLa cells were maintained in high-glucose Dulbecco's Modified Eagle's Medium (Hyclone) supplemented with $10 \%$ fetal bovine serum, $100 \mathrm{U} / \mathrm{mL}$ penicillin, and $100 \mu \mathrm{g} / \mathrm{mL}$ streptomycin under standard cell culture condition $\left(37^{\circ} \mathrm{C}\right.$, $5 \% \mathrm{CO}_{2} / 95 \%$ air). For both HepG2 and HeLa, the cells were seeded at a density of $\sim 5-6 \times 10^{3}$ cells per well in a 96-well culture plate each. After being in culture overnight, the cells were treated with free DOX and DOX-s-(PLAMA-b-PSBMA)-bPNIPAM at a range of designated concentrations for $24 \mathrm{~h}$. The CCK-8 cell viability assay was performed according to the manufacturer's instructions (Dojindo, Kumamoto, Japan).

\section{Cellular uptake of DOX-loaded nanogels}

Cellular uptake of DOX-s-(PLAMA-b-PSBMA)-b-PNIPAM by HeLa and HepG2 cells was examined. Cells were seeded in confocal microscopic dishes at a density of $\sim 2-3 \times 10^{5}$ cells per dish and cultured overnight. The cells were then exposed to free DOX and DOX-s-(PLAMA-b-PSBMA)-b-PNIPAM with a final DOX concentration of $5 \mu \mathrm{g} / \mathrm{mL}$ and incubated at $37^{\circ} \mathrm{C}$ for 2 or $6 \mathrm{~h}$. Then the cells were rinsed with PBS and stained with DAPI $(1 \mu \mathrm{g} / \mathrm{mL})$ for $5 \mathrm{~min}$. DOX uptake was examined under a confocal laser scanning microscope (TCS, SP8; Leica, Heidelberg, Germany).

To quantify the uptake of DOX by the tumor cells, free DOX and DOX-s-(PLAMA-b-PSBMA)-b-PNIPAM with a final DOX concentration of $5 \mu \mathrm{g} / \mathrm{mL}$ were incubated with HepG2 or HeLa $\left(\sim 1 \times 10^{5}\right)$ cells for 2 or $6 \mathrm{~h}$, following which cellular fluorescent intensity was measured by flow cytometry. Intracellular DOX level of each sample was presented as average fluorescent intensity.

\section{Results and discussion Synthesis of star polymer nanogels}

The chemical structures and compositions of LAMA, PLAMA, PLAMA-b-PSBMA, and s-(PLAMA-b-PSBMA)b-PNIPAM were determined by ${ }^{1} \mathrm{H}$ NMR and GPC. As shown in Figure 1, all characteristic signals of LAMA could be observed as previously reported, ${ }^{28}$ with the PLAMA, PLAMA-b-PSBMA, and s-(PLAMA-b-PSBMA)-b-PNIPAM segments clearly shown. After polymerization, the characteristic peaks that correspond to vinyl within the LAMA unit (at 5.75 and $6.25 \mathrm{ppm}$ ) disappeared, indicating successful conjugation of LAMA to PLAMA. Peaks that correspond to $\mathrm{C}\left(\mathrm{CH}_{3}\right) \mathrm{COOCH}_{2} \mathrm{CH}_{2} \mathrm{~N}\left(\mathrm{CH}_{3}\right)_{2} \mathrm{CH}_{2} \mathrm{CH}_{2} \mathrm{CH}_{2} \mathrm{SO}_{3}$ repeated methylene ${ }^{+} \mathrm{N}\left(\mathrm{CH}_{3}\right)_{2},{ }^{+} \mathrm{NCH}_{2}$ and $-\mathrm{CH}_{2} \mathrm{CH}_{2}$ at $3.4 \mathrm{ppm}$, $3.2 \mathrm{ppm}$, and $2.3 \mathrm{ppm}$ of the PSBMA also indicate successful polymerization. Moreover, the repeating units of PLAMA, PSBMA, and PNIPAM could be calculated by comparing the integral areas between characteristic peaks of the NIPAM unit at $2.6 \mathrm{ppm}\left(-\mathrm{N}-\mathrm{CH}-\left(\mathrm{CH}_{3}\right)_{2}\right)$, LAMA unit at $4.5 \mathrm{ppm}$, and the SBMA unit at $3.4 \mathrm{ppm}\left(-\mathrm{N}-\left(\mathrm{CH}_{3}\right)_{2}\right)$. Consistent with ${ }^{1} \mathrm{H}$ NMR results, molecular weight distribution (PDI) estimated by GPC of polymers PLAMA, PLAMA-b-PSBMA, and s-(PLAMA-b-PSBMA)-b-PNIPAM were within the same range and the details of these are listed in Table 1 . The narrow distribution of PDI for all the obtained PLAMA, PLAMA-bPSBMA, and s-(PLAMA-b-PSBMA)-b-PNIPAM polymers indicates that the polymerization process was well controlled and the purification methods were effective.

TEM images shown in Figure 2 reveal that the s-(PLAMAb-PSBMA)-b-PNIPAM and DOX-s-(PLAMA-b-PSBMA)b-PNIPAM nanogels were spherical in shape and relatively uniform. The average size of nanogels was $\sim 100-120 \mathrm{~nm}$. 


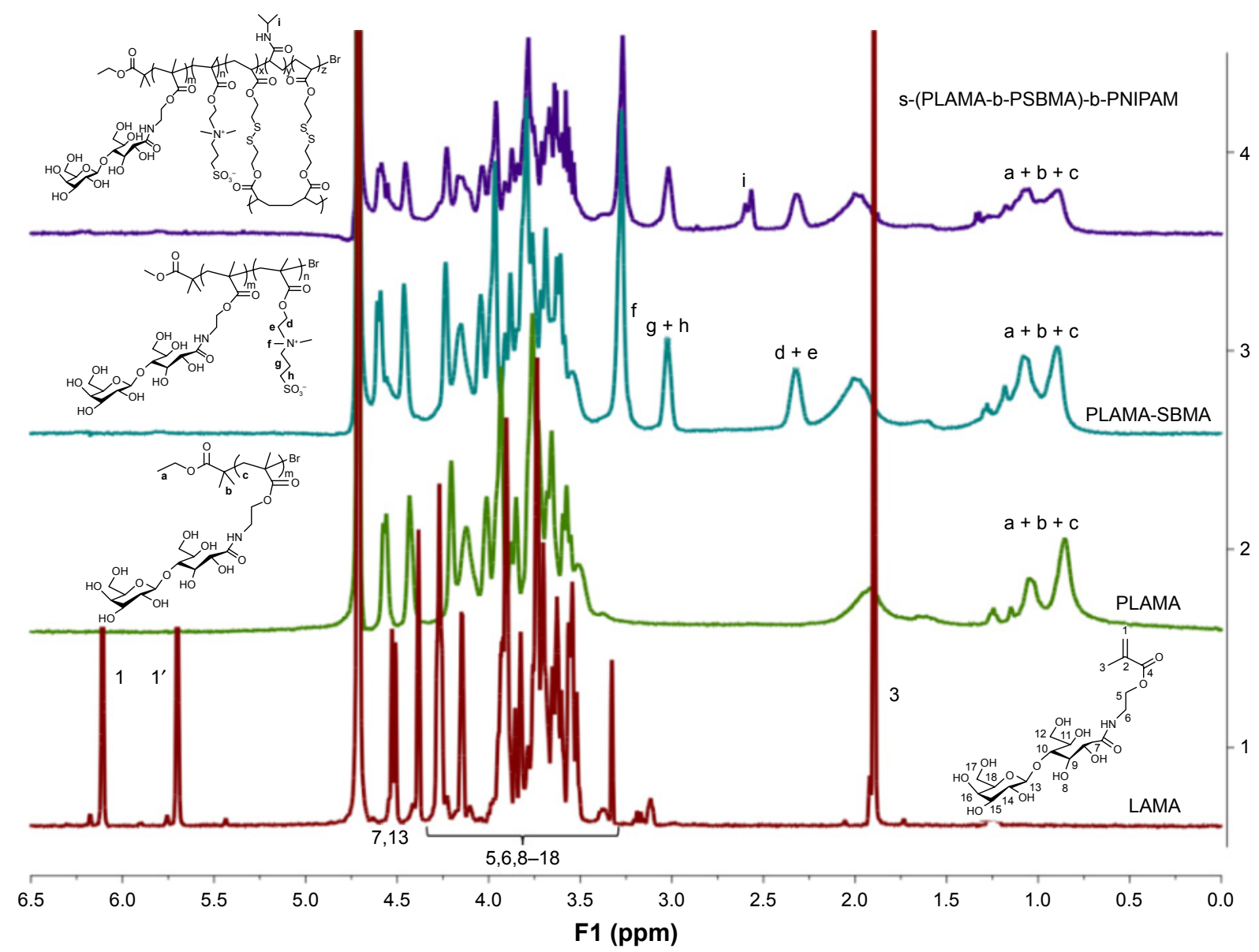

Figure I 'H NMR spectra of PLAMA, PLAMA-b-PSBMA, and s-(PLAMA-b-PSBMA)-b-PNIPAM were characterized by 'H NMR. 'H NMR was performed on a Bruker 400 $\mathrm{MHz}$ spectrometer using $\mathrm{D}_{2} \mathrm{O}$ as solvent.

Abbreviations: NMR, nuclear magnetic resonance; PLAMA, poly(2-lactobionamidoethyl methacrylamide); PSBMA, poly(sulfobetaine methacrylate); PNIPAM, poly(Nisopropylacrylamide).

However, the size of the nanogels detected by TEM was smaller than that determined by DLS (Table 2), likely due to shrinkage of nanoparticles during the drying process required prior to TEM. By contrast, in the presence of $10 \mathrm{mM} \mathrm{GSH}$, the nanogels fused and the average size decreased from $\sim 120$ to $\sim 70 \mathrm{~nm}$ (Figure 2C), as a result of GSH-induced disulfide bond breakage and polymer backbone dissolving. This rapid

Table I Characterization of the copolymers

\begin{tabular}{|c|c|c|c|c|c|c|}
\hline \multirow[t]{2}{*}{ Sample } & \multicolumn{3}{|c|}{ Composition } & \multirow[t]{2}{*}{$M_{n}{ }^{a}$} & \multirow[t]{2}{*}{$M_{n}^{b}$} & \multirow[t]{2}{*}{ PDI } \\
\hline & LAMA & SBMA & NIPAM & & & \\
\hline PLAMA $_{28}$ & 28 & 0 & 0 & 13,199 & 11,370 & 1.072 \\
\hline PLAMA $_{28}$-b-PSBMA 11 & 32 & II & 0 & 16,094 & 13,354 & 1.145 \\
\hline $\begin{array}{l}\text { s-(PLAMA }{ }_{28} \text {-b- } \\
\text { PSBMA )-b-PNIPAM }\end{array}$ & 32 & 11 & 22 & 18,583 & 15,763 & 1.320 \\
\hline
\end{tabular}

Notes: ${ }^{a}$ Molecular weight $\left(M_{n}\right)$ was determined by 'H NMR; ${ }^{b} M_{n}$ and PDI were measured by GPC.

Abbreviations: PDI, polydispersity index; PLAMA, poly(2-lactobionamidoethyl methacrylamide); PSBMA, poly(sulfobetaine methacrylate); PNIPAM, poly(N-isopropylacrylamide); NMR, nuclear magnetic resonance; GPC, gel permeation chromatography.
GSH-triggered nanogel disintegration enables specific drug release in tumor cells where reducing environment with high GSH concentration is characterized.

\section{Phase transition temperatures of $s$-(PLAMA-b-PSBMA)-b-PNIPAM nanogels in aqueous solution}

PolyNIPAM is the most widely studied thermo-responsive polymer that undergoes sharp phase transition at a LCST of $32^{\circ} \mathrm{C}$. By contrast, polySBMA, one example of zwitterionic polymers, exhibits an UCST in aqueous solution between $16^{\circ} \mathrm{C}$ and $18^{\circ} \mathrm{C}$, which indicates that the homopolymers in a dilute aqueous solution elicit a dramatic change in absorbance..$^{29}$ It has been reported previously that solubility of a similarly conjugated thermo-responsive copolymer occurs between the LCST and UCST values of the two polymer segments, as a result of intermolecular and intramolecular electrostatic interactions within the zwitterionic 

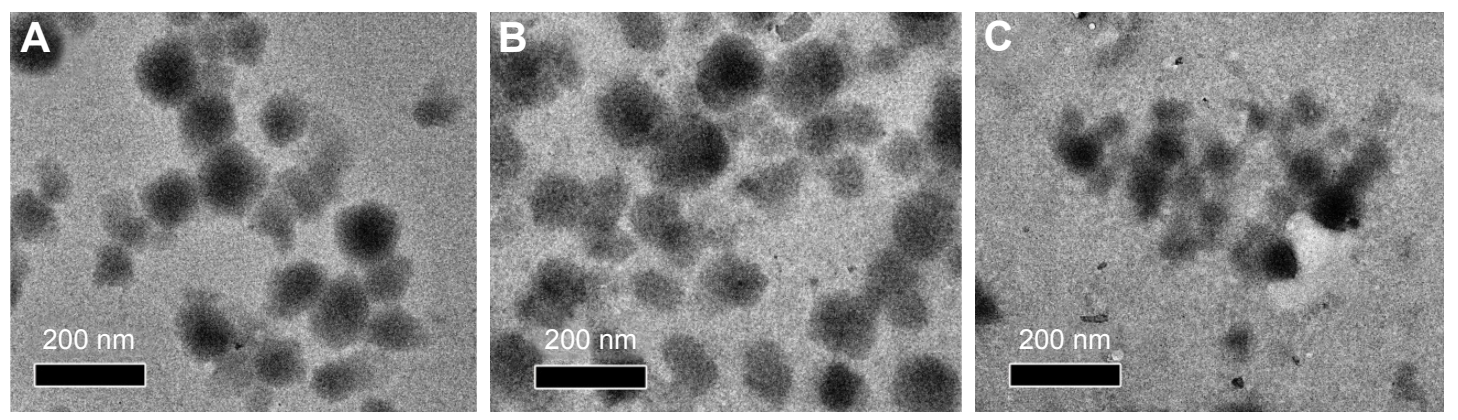

Figure 2 TEM images of s-(PLAMA-b-PSBMA)-b-PNIPAM nanogels (A), DOX-s-(PLAMA-b-PSBMA)-b-PNIPAM nanogels (B), and degradation of s-(PLAMA-b-PSBMA)-bPNIPAM nanogels after addition of $10 \mathrm{mM}$ GSH for $2 \mathrm{~h}(\mathbf{C})$.

Abbreviations: TEM, transmission electron microscope; PLAMA, poly(2-lactobionamidoethyl methacrylamide); PSBMA, poly(sulfobetaine methacrylate); PNIPAM, poly(N-isopropylacrylamide); DOX, doxorubicin; GSH, glutathione.

sulfobetaine groups of the SBMA segments, as well as the intramolecular hydrophobic interactions of nonionic isopropyl groups within the NIPAM segments. ${ }^{30}$ Consistent with previous reports, at temperatures below the UCST of PSBMA, s-(PLAMA-b-PSBMA)-b-PNIPAM nanogels exist as a collapsed coil and precipitate in water due to strong electrostatic of the zwitterionic groups. At temperatures above the LCST of PNIPAM, the PNIPAM chains become more hydrophobic and the hydrogen bonds formed with water molecules weaken, resulting in collapse of the polyNIPAM coils and polymer conjugation. As shown in Figure 3, the s-(PLAMA-b-PSBMA)-b-PNIPAM nanogels are soluble over a temperature range of $20^{\circ} \mathrm{C}-35^{\circ} \mathrm{C}$ (low absorbance), and the increase of absorbance was observed at temperatures either higher than $35^{\circ} \mathrm{C}$ or lower than $20^{\circ} \mathrm{C}$. The LCST of the PNIPAM-PSBMA copolymer increased because both PLAMA and PSBMA block are hydrophilic polymers. In addition, phase transition of s-(PLAMA-b-PSBMA)-b-PNIPAM nanogels in aqueous solution was studied as a function of temperature to investigate the effect of varied cross-linker ratios of copolymers on both UCST and LCST. Data in Table 2 show an inverse correlation between cross-linker ratio and UCST values from $18.6^{\circ} \mathrm{C}$ to $16.9^{\circ} \mathrm{C}$. By contrast, increase in cross-linker ratio correlates to increased LCST values from $39.1^{\circ} \mathrm{C}$ to $36.5^{\circ} \mathrm{C}$, suggesting that the increase of PNIPAM block cross-linking contributes to the insoluble collapsed coil state of the PNIPAM-PSBMA copolymers.

\section{Drug loading and releasing property of star polymer nanogel}

Drug release behaviors of s-(PLAMA-b-PSBMA)b-PNIPAM nanogels in response to different stimuli were investigated and the results are shown in Figure 4. The LC of nanogels (BAC 0.5\%) was lower than that of nanogels (BAC $1.0 \%$ ) and nanogels (BAC 2.0\%), indicating that the hydrophobic cross-linking chain plays an important role in this process. The temperature sensitive nanogels remain soluble as the temperature is below its LCST and after the nanogels start circulating to the malignant hyperthermal tissue, the nanogels precipitate, and the drug is released. Given such sensitivity to temperature changes, these nanogels would be employed for local tumor hyperthermia therapy. Among these incubation conditions, $37^{\circ} \mathrm{C}$ and $40^{\circ} \mathrm{C}$ represent the normal physiological temperature and hyperthermia treatment temperature, respectively. To investigate the influence of temperature changes on drug release, the experiments were conducted in buffer solutions at $\mathrm{pH} 6.5$ (to mimic the physiological pH of endosome) at different temperatures. It was observed that the

Table 2 Characterization and properties of the nanoparticles

\begin{tabular}{|c|c|c|c|c|c|c|c|}
\hline Sample & BAC wt\% & LCST $^{a}$ & UCST $^{\mathrm{b}}$ & Diameter ${ }^{c}$ & PDI $^{d}$ & EE\% & LC $\%$ \\
\hline$a:\left(\right.$ PLAMA $_{28}$-b-PSBMA 11 )-b-PNIPAM ${ }_{22}$ & $0.5 \%$ & 39.1 & 18.6 & $214.6 \mathrm{~nm}$ & 0.161 & $56.4 \pm 7.6$ & $8.7 \pm 0.9$ \\
\hline b:(PLAMA ${ }_{28}$-b-PSBMA 11$)$-b-PNIPAM ${ }_{22}$ & $1.0 \%$ & 38.2 & 17.7 & $207.2 \mathrm{~nm}$ & 0.225 & $61.2 \pm 8.3$ & $9.4 \pm 1.3$ \\
\hline$c:\left(\right.$ PLAMA $_{28}-$ b-PSBMA 11$)$-b-PNIPAM ${ }_{22}$ & $2.0 \%$ & 36.5 & 16.9 & $189.2 \mathrm{~nm}$ & 0.243 & $67.8 \pm 8.7$ & $10.6 \pm 1.1$ \\
\hline
\end{tabular}

Notes: ${ }^{a, b}$ UCST and LCST were determined by reading the absorbance at $550 \mathrm{~nm}$ on a UV-visible spectrophotometer. ${ }^{\mathrm{c}, \mathrm{d}} \mathrm{Average}$ diameter of nanogels and polydispersity index were determined by a Zetasizer Nano ZS instrument at $25^{\circ} \mathrm{C}$. The EE and drug LC are defined as follows: LC $(\%)=($ Weight of DOX in nanogels $/$ Weight of nanogels) $\times 100 \%$ EE $(\%)=($ Weight of DOX in nanogels/Weight of DOX in feed $) \times 100 \%$.

Abbreviations: BAC, N,N'-bis(acryloyl)cystamine; LCST, lower critical solution temperature; UCST, upper critical solution temperature; PLAMA, poly(2lactobionamidoethyl methacrylamide); PSBMA, poly(sulfobetaine methacrylate); PNIPAM, poly(N-isopropylacrylamide); EE, entrapment efficiency; LC, loading capacity; DOX, doxorubicin. 


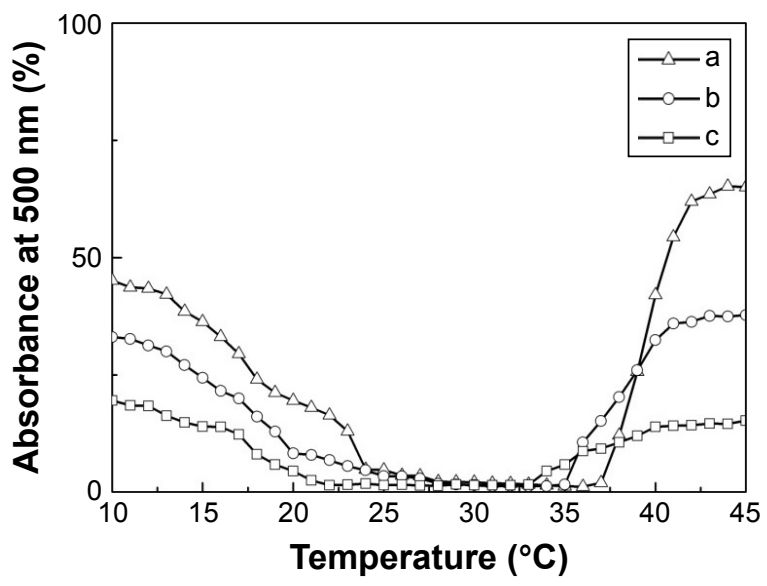

Figure 3 UCST and LCST curves for s-(PLAMA-b-PSBMA)-b-PNIPAM nanogels with different cross-linking degree.

Notes: a, (PLAMA ${ }_{28}$-b-PSBMA ${ }_{11}$ )-b-PNIPAM ${ }_{22}$ at BAC 0.5\%; b, (PLAMA P $_{28}$-b-PSBMA $\left.A_{11}\right)$ b-PNIPAM ${ }_{22}$ at BAC 1.0\%; and c, (PLAMA ${ }_{28}$-b-PSBMA 11 )-b-PNIPAM 22 at BAC $2.0 \%$. Abbreviations: UCST, upper critical solution temperature; LCST, lower critical solution temperature; PLAMA, poly(2-lactobionamidoethyl methacrylamide); PSBMA, poly(sulfobetaine methacrylate); PNIPAM, poly(N-isopropylacrylamide).

DOX release rate increased as the temperature decreased from $40^{\circ} \mathrm{C}$ to $37^{\circ} \mathrm{C}$ and $30^{\circ} \mathrm{C}$. Drug release in response to environmental redox changes was also investigated in parallel to assess whether the lactose-functionalized nanogel could be degraded by GSH. The release rate of DOX was significantly enhanced in the presence of $10 \mathrm{mM}$ GSH regardless of environmental temperature conditions, showing a maximum of $80 \%$ drug release within $24 \mathrm{~h}$. The accelerated drug
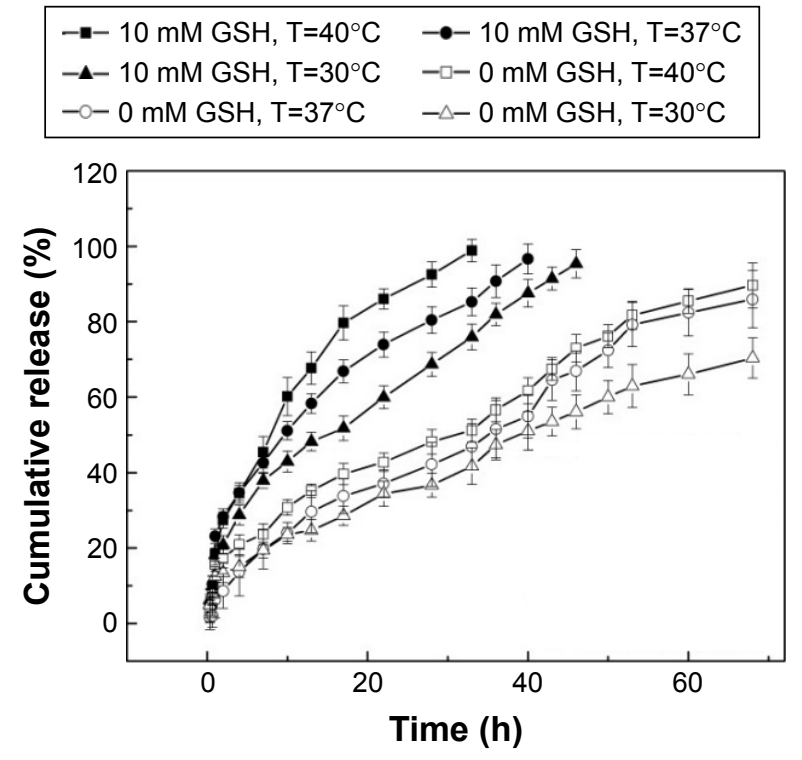

Figure 4 In vitro release profiles of DOX from the DOX-loaded s-(PLAMA-bPSBMA)-b-PNIPAM nanogels under different conditions. $P<0.0510 \mathrm{mM} \mathrm{GSH}$, $\mathrm{T}=40^{\circ} \mathrm{C}$ vs $10 \mathrm{mM} \mathrm{GSH}, \mathrm{T}=37^{\circ} \mathrm{C} ; P<0.0510 \mathrm{mM}$ GSH vs $0 \mathrm{mM} \mathrm{GSH} ; P<0.010 \mathrm{mM}$ $\mathrm{GSH}, \mathrm{T}=40^{\circ} \mathrm{C}$ vs $0 \mathrm{mM} \mathrm{GSH}, \mathrm{T}=30^{\circ} \mathrm{C} ; P<0.050 \mathrm{mM} \mathrm{GSH}, \mathrm{T}=37^{\circ} \mathrm{C}$ vs $0 \mathrm{mM} \mathrm{GSH}$, $\mathrm{T}=30^{\circ} \mathrm{C}$ via one-way ANOVA.

Abbreviations: DOX, doxorubicin; PLAMA, poly(2-lactobionamidoethyl methacrylamide); PSBMA, poly(sulfobetaine methacrylate); PNIPAM, poly(N-isopropylacrylamide); GSH, glutathione; ANOVA, analysis of variance. release from the redox-degradable nanogels, to some extent, demonstrates that the rate of drug release was dependent on the cleavage rate of disulfide bond within the PNIPAM core. To further elucidate the in vitro thermo-responsive drug release mechanism of DOX-nanogels, we chose $(0 \mathrm{mM}$ $\mathrm{GSH}, 37^{\circ} \mathrm{C}$ ) release profile as an example; the data were analyzed using the zero-order equation, Higuchi equation, and Ritger-Peppas equation models. The fitting results are (zero exponential: $\mathrm{Q}=0.012 * \mathrm{t}+0.139, r^{2}=0.926$; RitgerPeppas) $\ln Q=1.815^{*} \ln t+4.425, r^{2}=0.95896$; Higuchi: $\mathrm{Q}=2.65 * \mathrm{t} 1 / 2+7.277, r^{2}=0.8415$. For in vitro GSH-responsive drug release mechanism of DOX-nanogels, we chose $\left(10 \mathrm{mM} \mathrm{GSH}, 37^{\circ} \mathrm{C}\right.$ ) release profile as an example; the data were analyzed using the zero-order equation, Higuchi equation, and Ritger-Peppas equation models (zero exponential: $\mathrm{Q}=2.041 * \mathrm{t}+22.083, r^{2}=0.9016$; RitgerPeppas: $\ln \mathrm{Q}=0.542 * \ln \mathrm{t}-1.952, r^{2}=0.9237$; Higuchi: $\left.\mathrm{Q}=0.148 * \mathrm{t} 1 / 2+0.031, r^{2}=0.9863\right)$. These results revealed that the in vitro release profile of DOX-nanogels with GSH followed the Higuchi equation $\left(r^{2}=0.9863\right)$. These indicate that DOX release was the synergistic effect of nanogel degradation and drug diffusion. ${ }^{31,32}$ The dual-sensitivity-triggered DOX release behavior could be explained by the coordinated dual sensitivity.

\section{Assessment of hepatic targeting property and anti-tumor activity}

To evaluate the hepatic targeting ability of the nanogelbased delivery system and its cytotoxicity, human hepatoma HepG2 cells were used since the lactose groups are known to recognize the ASGP-R, a recycling endocytotic receptor often found overexpressed by HepG2. Human cervical carcinoma cells, HeLa, that do not express ASGP-R were used as negative control. The blank nanogel s-(PLAMA-b-PSBMA)b-PNIPAM showed very low cytotoxicity even at fairly high concentrations up to $10 \mathrm{mg} / \mathrm{mL}$ (Figure 5). The cytotoxicity of DOX and DOX-loaded s-(PLAMA-b-PSBMA)-bPNIPAM was measured by CCK-8 assay. HeLa and HepG2 cells were treated with free DOX or DOX-loaded nanogels at a series of DOX concentrations ranging from 0.001 to $10.0 \mu \mathrm{g} / \mathrm{mL}$ for 24 and $48 \mathrm{~h}$. As shown in Figure 6, the lactosylated nanogel exhibited lower activity in inhibiting proliferation of HepG2 cells in the presence of galactose because the presence of galactose would mask the lactosylated segment on the nanogel and disrupt the hepatomatargeting ability of the nanogel. ${ }^{6}$ By contrast, addition of galactose only affected the proliferation activity of HeLa cells moderately, confirming that the hepatoma-targeting property of the lactose-bearing nanogel was dependent 


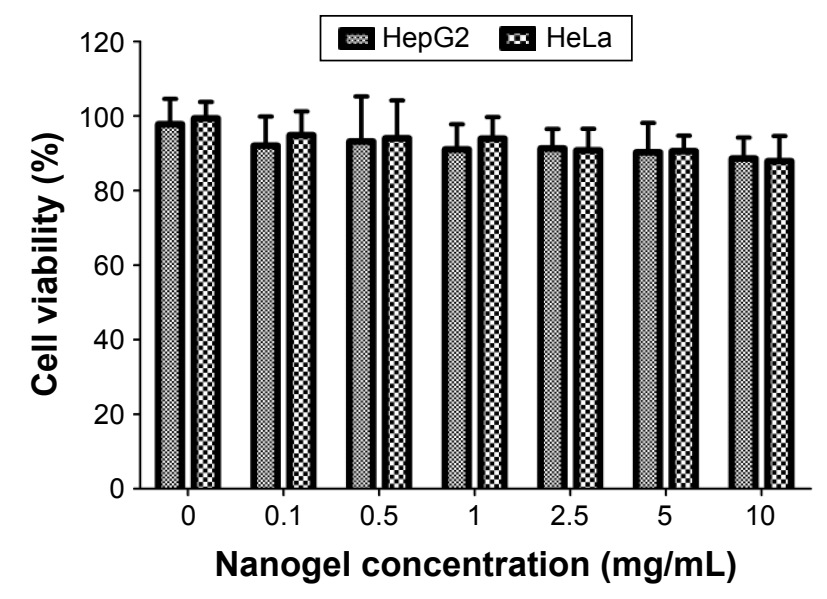

Figure 5 Cell viability of HeLa and HepG2 cells after exposure to the blank nanogels at various concentrations.

on ASGP-R recognition by the lactosylated moiety. As expected, the presence or absence of galactose did not affect the inhibiting activity of free DOX for HepG2 and HeLa cells.
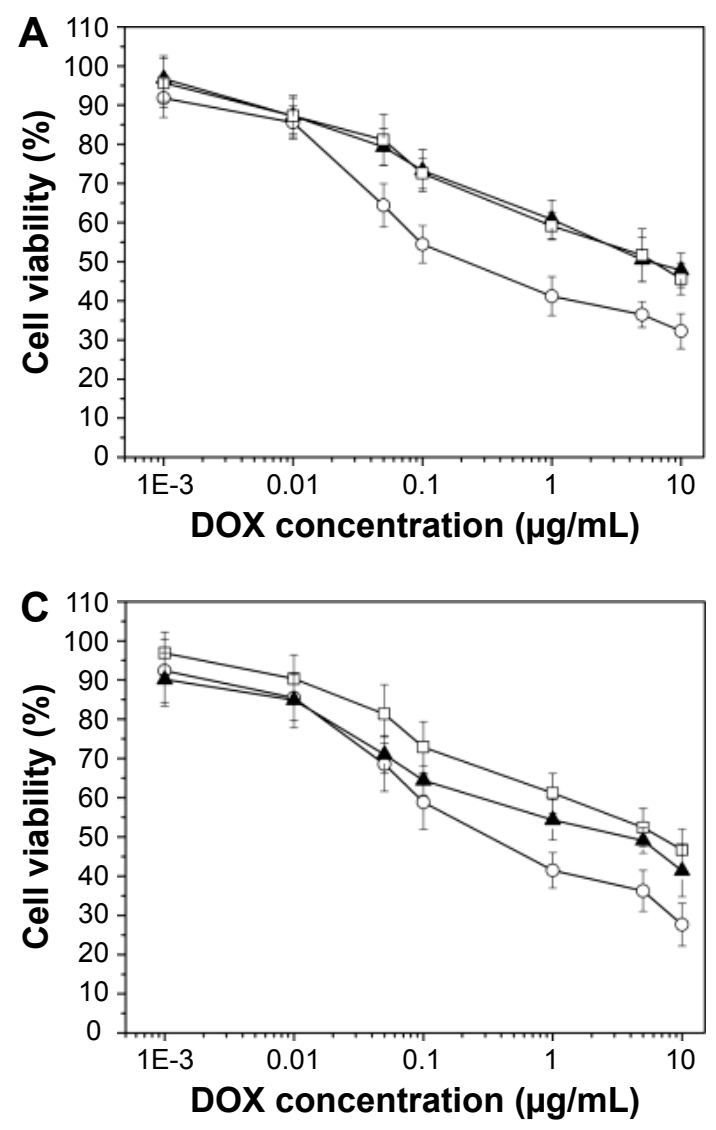

Indeed, DOX-loaded nanogels exhibited higher inhibitory effect on the proliferation of HepG2 cells that express ASGP-R compared to HeLa cells. Remarkably, the presence and absence of galactose dramatically affected the $\mathrm{IC}_{50}$ values of DOX-loaded nanogels (Table 3) (HepG2 cells: $4.46 \mu \mathrm{g} / \mathrm{mL}$ [without galactose] vs $7.51 \mu \mathrm{g} / \mathrm{mL}$ [with galactose] after incubation for $24 \mathrm{~h} ; 0.31 \mu \mathrm{g} / \mathrm{mL}$ [without galactose] vs $1.42 \mu \mathrm{g} / \mathrm{mL}$ [with galactose] after incubation for $48 \mathrm{~h}$ ); In comparison to HepG2 cells, whether there is a presence or absence of galactose, the $\mathrm{IC}_{50}$ values of lactosylated nanomedicine for HeLa cell are only slightly affected, as detailed in Table 3. Changes in $\mathrm{IC}_{50}$ values by addition of galactose in HepG2 cells indicate ASGP-R-specific binding of the lactosylated segment. It is noteworthy that the $\mathrm{IC}_{50}$ of lactosylated nanogels was close to that of free DOX $(0.21 \mu \mathrm{g} / \mathrm{mL})$, suggesting excellent drug release and liver-specific targeting. Furthermore, the hepatic-specific targeting property of the lactosylated nanogels was confirmed by results obtained from HeLa cells. The cytotoxicity
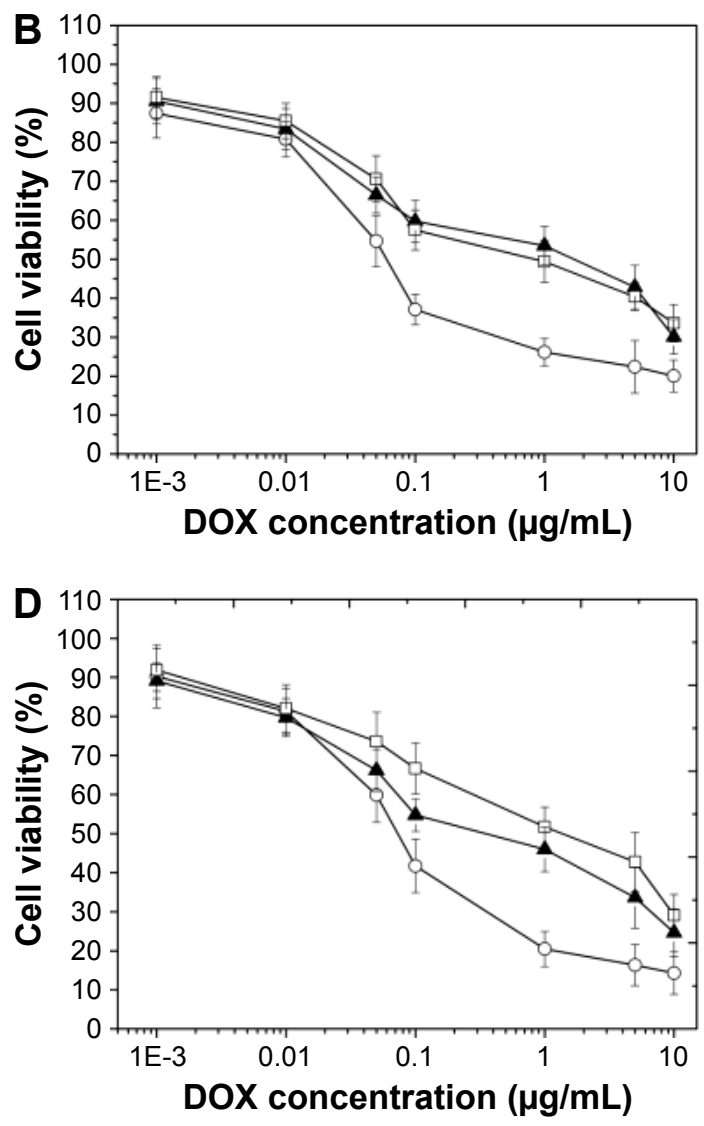

$\longrightarrow-a-b \quad a-c$

Figure 6 Proliferation inhibition of HeLa (A, 24 h; B, 48 h) and HepG2 (C, 24 h; D, 48 h) cells by incubation with DOX (a) or DOX-loaded-(PLAMA-b-PSBMA)-b-PNIPAM nanogels (b, c) at various DOX concentrations. The incubations were in the absence (b) or presence of $20.0 \mathrm{mM}$ galactose (c). (A) HeLa (24 h): $P<0.0 \mathrm{I}$ a and b vs c; (C and $\mathbf{D})$ HepG2: $P<0.05$ a vs c via one-way ANOVA.

Abbreviations: DOX, doxorubicin; PLAMA, poly(2-lactobionamidoethyl methacrylamide); PSBMA, poly(sulfobetaine methacrylate); PNIPAM, poly(N-isopropylacrylamide); ANOVA, analysis of variance. 
Table $3 I_{50}$ values of nanomedicines from DOX-loaded nanogels after incubation with HepG2 and HeLa cells in the absence or presence of $20.0 \mathrm{mM}$ galactose for 24 and $48 \mathrm{~h}$

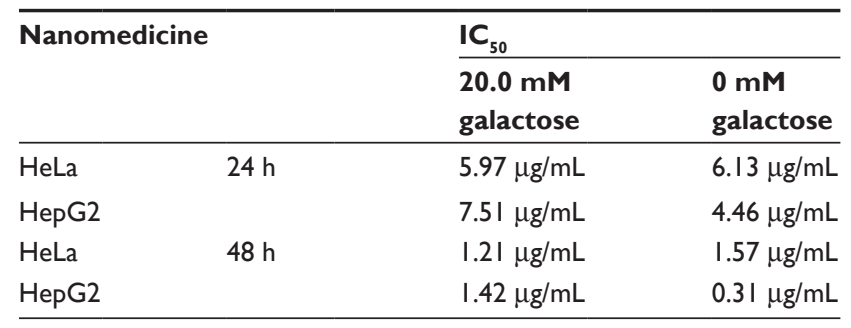

Abbreviation: DOX, doxorubicin.

of s-(PLAMA-b-PSBMA)-b-PNIPAM for HepG2 cells in the absence of galactose was significantly higher than that in the presence of galactose, which could be attributable to enhanced endocytosis. Thus, to demonstrate enhanced binding ability of lactosylated nanogels to HepG2 cells, the receptor-mediated cellular uptake and intracellular DOX release were evaluated by CLSM and flow cytometry.

HepG2 and HeLa cells were incubated with free DOX or DOX-s-(PLAMA-b-PSBMA)-b-PNIPAM nanogels for 2 and $6 \mathrm{~h}$. It could be observed that the presence of galactose significantly affected intracellular uptake of DOX-s-(PLAMAb-PSBMA)-b-PNIPAM nanogels in HepG2 cells after $2 \mathrm{~h}$ of incubation (Figure 7), whereas no difference was detectable in HeLa cells regardless of galactose (Figure 7), highlighting the facilitative role of lactose moiety on hepatoma targeting. Flow cytometry was also employed to investigate the effect and mechanisms of DOX-s-(PLAMA-b-PSBMA)-bPNIPAM nanogels on intracellular DOX uptake. Consistent with CLSM data, the results indicate that addition of
A
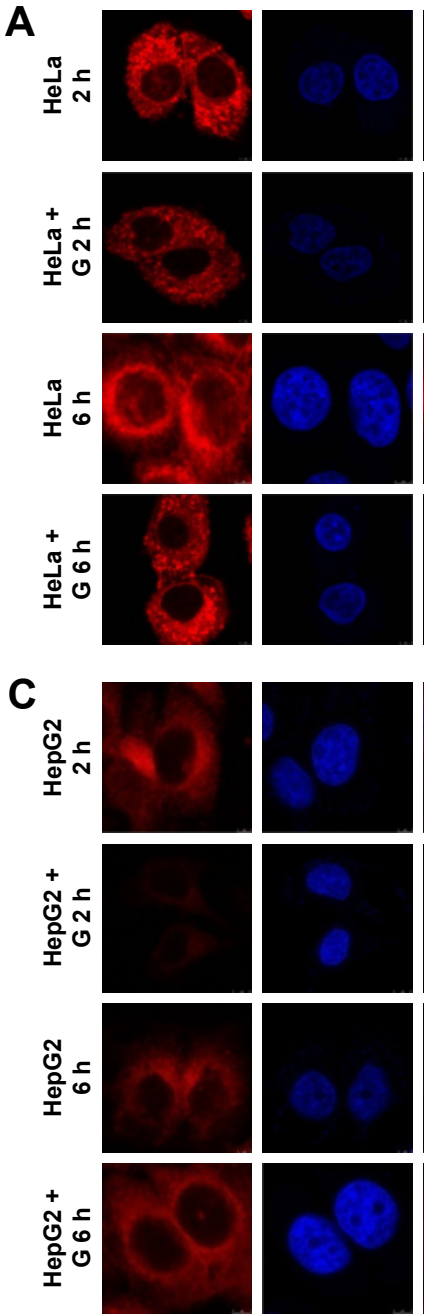
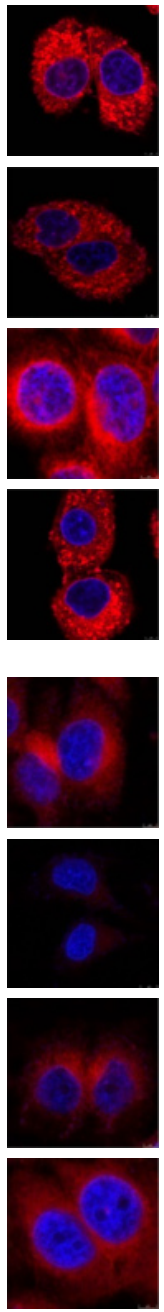
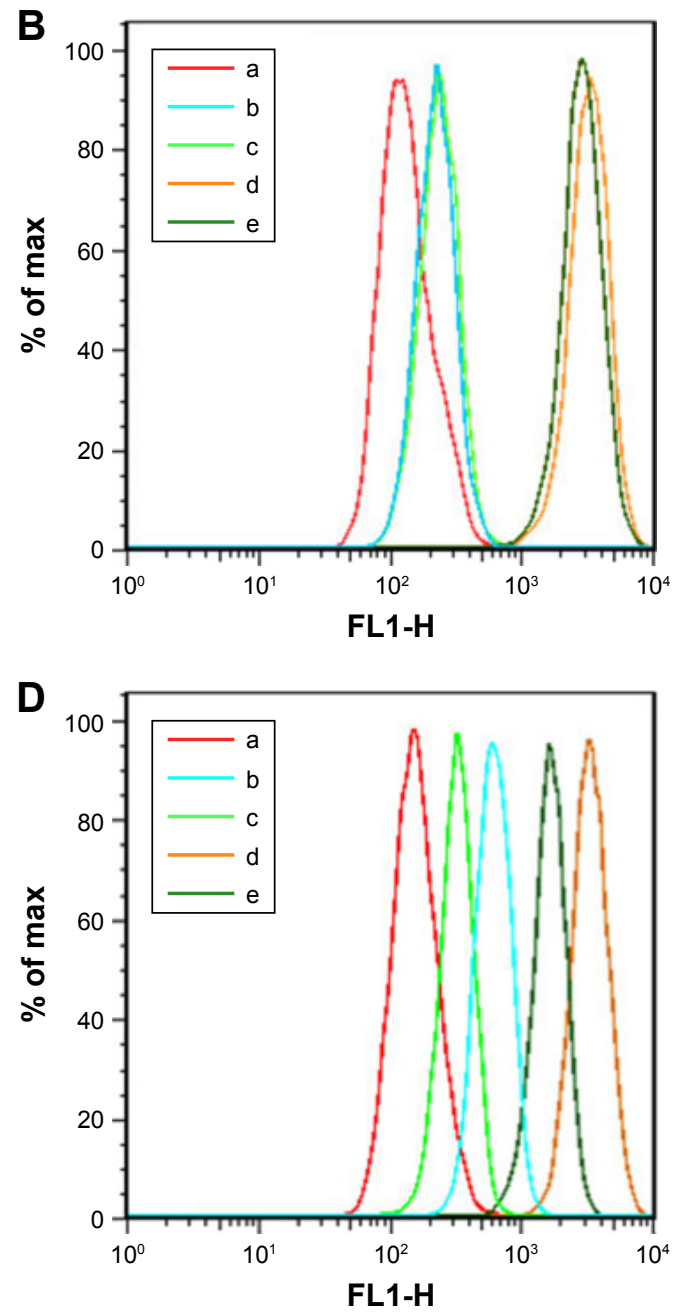

Figure 7 CLSM images of HeLa (A) and HepG2 (C) cells incubated with DOX-s-(PLAMA-b-PSBMA)-b-PNIPAM nanogels $(5 \mu \mathrm{g} / \mathrm{mL})$ after 2 and $6 \mathrm{~h}$ in the presence or absence of galactose, respectively. For each panel, the images from left to right show DOX fluorescence in cells (red), cell nuclei stained by DAPI (blue), and overlays of the two images. Flow cytometric profiles of HeLa (B) and HepG2 (D) cells after incubation with DOX-loaded s-(PLAMA-b-PSBMA)-b-PNIPAM at 2 h (b, absence of 20.0 mM galactose; c, presence of $20.0 \mathrm{mM}$ galactose) and $6 \mathrm{~h}$ (d, absence of $20.0 \mathrm{mM}$ galactose; e, presence of $20.0 \mathrm{mM}$ galactose); cells incubated with PBS were used as control (a). Data are presented as mean \pm standard deviation $(n=3)$.

Abbreviations: DOX, doxorubicin; PLAMA, poly(2-lactobionamidoethyl methacrylamide); PSBMA, poly(sulfobetaine methacrylate); PNIPAM, poly(N-isopropylacrylamide); PBS, phosphate-buffered saline. 


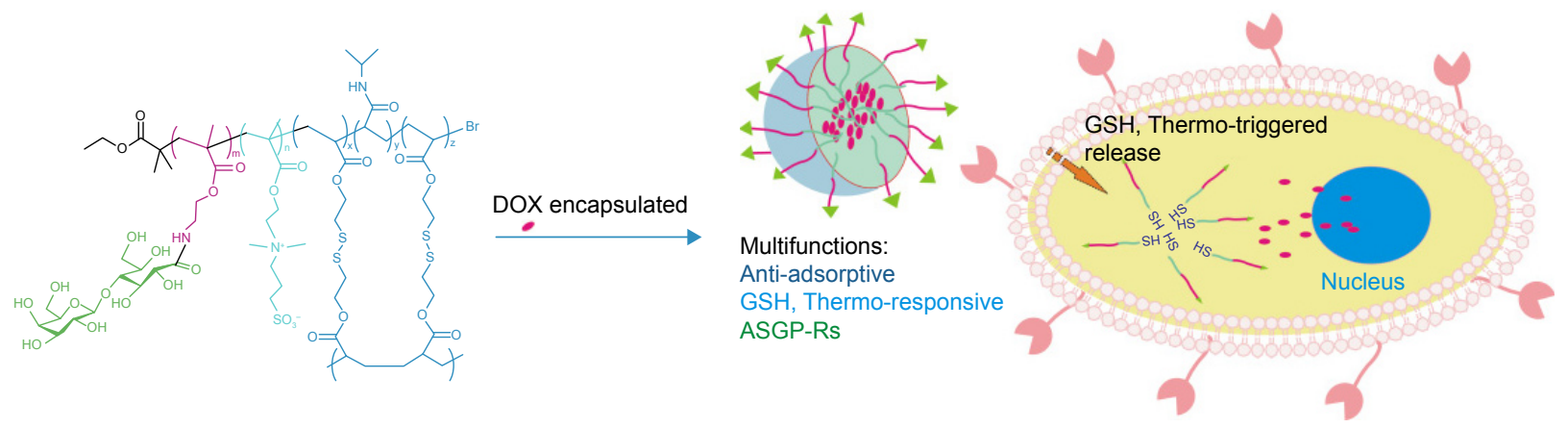

Scheme 2 Illustration of star-shaped glycopolymer s-(PLAMA-b-PSBMA)-b-PNIPAM nanogels for ASGP-R-mediated endocytosis and efficient thermo- and GSH-triggered release.

Abbreviations: PLAMA, poly(2-lactobionamidoethyl methacrylamide); PSBMA, poly(sulfobetaine methacrylate); PNIPAM, poly(N-isopropylacrylamide); ASGP-R, asialoglycoprotein receptor; GSH, glutathione; DOX, doxorubicin.

galactose, which blocks binding of nanogel and ASGP-R since galactose also binds to the ASGP-R of HepG2 cells, inhibits endocytosis of the lactosylated nanogels, shown by reduction in DOX fluorescence signals of the galactose + nanogel groups compared to nanogel-only groups (Figure 7B and D [b and c, 2 h; $d$ and e, 6 h]). No detectable difference regarding DOX fluorescence signals was observed in HeLa cells with the addition of galactose, suggesting that the selectivity preference of hepatocytes for lactosylated nanomedicine was mediated by the ASGP-R.

\section{Conclusion}

In the present study, we prepared thermo- and redox-sensitive star-shaped nanogels. The star-shaped nanogels consist of a thermo- and redox-sensitive core that is composed of disulfide cross-linked PNIPAM, and "arm" segments of zwitterionic PSBMA and PLAMA. In vitro DOX release from nanogels was accelerated in conditions that mimic malignant cell microenvironment characterized as hyperthermal with high GSH concentration. Our results also demonstrate that the (PLAMA-b-PSBMA)-b-PNIPAM nanogels enhanced and selected DOX delivery in HepG2 cells as the result of lactose residue-specific binding with the ASGP-R (Scheme 2). In summary, our data indicate that the lactose-functionalized multi-responsive nanogels could be potentially used for drug delivery against hepatic cancers.

\section{Acknowledgment}

This study was financially supported by National Natural Science Foundation of China (81301309, 31300732), Natural Science Foundation of Tianjin (16JCQNJC14200), Tianjin International Science and Technology Cooperation Projects (15RCGFSY00146), Nankai University Doctoral Science Innovation Foundation (68150003), and Program for Innovative Research Team in Peking Union Medical College.

\section{Disclosure}

The authors report no conflicts of interest in this work.

\section{References}

1. Yin Q, Shen J, Zhang Z, Yu H, Li Y. Reversal of multidrug resistance by stimuli-responsive drug delivery systems for therapy of tumor. Adv Drug Deliv Rev. 2013;65(13-14):1699-1715.

2. Elsabahy M, Wooley KL. Design of polymeric nanoparticles for biomedical delivery applications. Chem Soc Rev. 2012;41(7):2545-2561.

3. Dehaini D, Fang RH, Zhang L. Biomimetic strategies for targeted nanoparticle delivery. Bioeng Translation Med. 2016;1(1):30-46.

4. Luo HC, Li N, Yan L, et al. Ligand-based targeted therapy: a novel strategy for hepatocellular carcinoma. Int J Nanomedicine. 2017;12: 1085-1096.

5. Zhang YN, Poon W, Tavaresa AJ, McGilvray ID, Chan WC. Nanoparticle-liver interactions: cellular uptake and hepatobiliary elimination. J Control Release. 2016;240:332-348.

6. Ding J, Xiao C, Li Y, et al. Efficacious hepatoma-targeted nanomedicine self-assembled from galactopeptide and doxorubicin driven by twostage physical interactions. J Control Release. 2013;169(3):193-203.

7. Alvarez-Berríos MP, Vivero-Escoto JL. In vitro evaluation of folic acid-conjugated redox-responsive mesoporous silica nanoparticles for the delivery of cisplatin. Int J Nanomedicine. 2016;11:6251-6265.

8. Li Y, Liu R, Yang J, et al. Enhanced retention and anti-tumor efficacy of liposomes by changing their cellular uptake and pharmacokinetics behavior. Biomaterials. 2015;41:1-14.

9. Wang Y, Liu X, Liu G, et al. Novel galactosylated biodegradable nanoparticles for hepatocyte-delivery of oridonin. Int J Pharm. 2016; 502(1-2):47-60.

10. Thao LQ, Lee C, Kim B, et al. Doxorubicin and paclitaxel co-bound lactosylated albumin nanoparticles having targetability to hepatocellular carcinoma. Colloids Surf B Biointerfaces. 2017;152:183-191.

11. Mohamed NK, Hamad MA, Hafez MZ, Wooley KL, Elsabahy M. Nanomedicine in management of hepatocellular carcinoma: challenges and opportunities. Int $J$ Cancer. 2017;140(7):1475-1484.

12. Li N, Luo HC, Yang C, et al. Cationic star-shaped polymer as an siRNA carrier for reducing MMP-9 expression in skin fibroblast cells and promoting wound healing in diabetic rats. Int J Nanomedicine. 2014;9: 3377-3387.

13. Syrett JA, Haddleton DM, Whittaker MR, Davis TP, Boyer C. Functional, star polymeric molecular carriers, built from biodegradable microgel/ nanogel cores. Chem Commun (Camb). 2011;47(5):1449-1451.

14. Wang D, Jin Y, Zhu X, Yan D. Synthesis and applications of stimuli-responsive hyperbranched polymers. Prog Polym Sci. 2017;64:114-153.

15. Oh JK, Siegwart DJ, Lee HI, et al. Biodegradable nanogels prepared by atom transfer radical polymerization as potential drug delivery carriers: synthesis, biodegradation, in vitro release, and bioconjugation. $J \mathrm{Am}$ Chem Soc. 2007;129(18):5939-5945. 
16. Mou Q, Ma Y, Zhu X, Yan D. A small molecule nanodrug consisting of amphiphilic targeting ligand-chemotherapy drug conjugate for targeted cancer therapy. J Control Release. 2016;230:34-44.

17. Zhao J, Babiuch K, Lu H, Dag A, Gottschaldt M, Stenzel MH. Fructose-coated nanoparticles: a promising drug nanocarrier for triplenegative breast cancer therapy. Chem Commun (Camb). 2014;50(100): $15928-15931$

18. Song EH, Manganiello MJ, Chow YH, et al. In vivo targeting of alveolar macrophages via RAFT-based glycopolymers. Biomaterials. 2012;33(28):6889-6897.

19. Kempe K, Xiang SD, Wilson P, et al. Engineered hydrogen-bonded glycopolymer capsules and their interactions with antigen presenting cells. ACS Appl Mater Interfaces. 2017;9(7):6444-6452.

20. Zhang Y, Chan JW, Moretti A, Uhrich KE. Designing polymers with sugar-based advantages for bioactive delivery applications. J Control Release. 2015;219:355-368.

21. Ahmed M, Narain R. The effect of molecular weight, compositions and lectin type on the properties of hyperbranched glycopolymers as nonviral gene delivery systems. Biomaterials. 2012;33(15):3990-4001.

22. Liu P, Sun Y, Wang Q, Sun Y, Li H, Duan Y. Intracellular trafficking and cellular uptake mechanism of mPEG-PLGA-PLL and mPEGPLGA-PLL-Gal nanoparticles for targeted delivery to hepatomas. Biomaterials. 2014;35(2):760-770.

23. Wang HX, Xiong MH, Wang YC, Zhu J, Wang J. N-acetylgalactosamine functionalized mixed micellar nanoparticles for targeted delivery of siRNA to liver. J Control Release. 2013;166(2):106-114.

24. Chen W, Zou Y, Meng F, et al. Glyco-nanoparticles with sheddable saccharide shells: a unique and potent platform for hepatoma-targeting delivery of anticancer drugs. Biomacromolecules. 2014;15(3): 900-907.
25. Lu C, Xing MM, Zhong W. Shell cross-linked and hepatocyte-targeting nanoparticles containing doxorubicin via acid cleavable linkage. Nanomedicine. 2011;7(1):80-87.

26. Wang J, Yang G, Guo X, Tang Z, Zhong Z, Zhou S. Redox-responsive polyanhydride micelles for cancer therapy. Biomaterials. 2014;35(9): 3080-3090.

27. Thapa B, Kumar P, Zeng H, Narain R. Asialoglycoprotein ReceptorMediated Gene Delivery to Hepatocytes Using Galactosylated Polymers. Biomacromolecules. 2015;16(9):3008-3020.

28. Narain R, Armes SP. Synthesis and aqueous solution properties of novel sugar methacrylate-based homopolymers and block copolymers. Biomacromolecules. 2003;4(6):1746-1758.

29. Cummings C, Murata H, Koepsel R, Russell AJ. Dramatically increased $\mathrm{pH}$ and temperature stability of chymotrypsin using dual block polymerbased protein engineering. Biomacromolecules. 2014;15(3):763-771.

30. Shih YJ, Chang Y, Deratani A, Quemener D. "Schizophrenic" hemocompatible copolymers via switchable thermoresponsive transition of nonionic/zwitterionic block self-assembly in human blood. Biomacromolecules. 2012;13(9):2849-2858.

31. Luo Y, Liu Z, Zhang X, et al. Effect of a controlled-release drug delivery system made of oleanolic acid formulated into multivesicular liposomes on hepatocellular carcinoma in vitro and in vivo. Int J Nanomedicine. 2016;11:3111-3129.

32. Serra L, Doménech J, Peppas NA. Drug transport mechanisms and release kinetics from molecularly designed poly(acrylic acid-g-ethylene glycol) hydrogels. Biomaterials. 2006;27(31):5440-5451.
International Journal of Nanomedicine

\section{Publish your work in this journal}

The International Journal of Nanomedicine is an international, peerreviewed journal focusing on the application of nanotechnology in diagnostics, therapeutics, and drug delivery systems throughout the biomedical field. This journal is indexed on PubMed Central, MedLine, CAS, SciSearch $®$, Current Contents ${ }^{\circledR} /$ Clinical Medicine,

\section{Dovepress}

Journal Citation Reports/Science Edition, EMBase, Scopus and the Elsevier Bibliographic databases. The manuscript management system is completely online and includes a very quick and fair peer-review system, which is all easy to use. Visit http://www.dovepress.com/ testimonials.php to read real quotes from published authors. 